\title{
CONSULTA ODONTOLÓGICA E DOENÇA SISTÊMICA: ANÁLISE DO CONHECIMENTO DOS CIRURGIÕES- DENTISTAS EM ITAJAÍ-SC
}

\author{
DENTAL VISITS AND SYSTEMIC DISORDERS: AN ANALYSIS \\ OF THE SURGEON-DENTISTS' KNOWLEDGE IN ITAJAÍ-SC
}

\author{
Maria Regina Kreuger \\ Nivaldo Murilo Diegoli \\ Rômulo D'Avila Pedrini \\ Vanessa Raup Porfírio \\ Francine da Silva
}

\section{RESUMO}

A anamnese é feita no primeiro contato entre o cirurgiãodentista e o paciente; as informações obtidas devem ser anotadas em uma ficha clínica. Em virtude do aumento no número de pacientes em diferentes faixas etárias necessitando de tratamentos diferenciados, observa-se a necessidade de padronização das fichas clínicas. Considerando-se a importância desta etapa do atendimento odontológico, desenvolveu-se uma pesquisa com o objetivo de verificar se a anamnese do paciente integra a rotina dos cirurgiõesdentistas $(\mathrm{CD})$, quais as doenças sistêmicas e de emergência mais encontradas no consultório e quais os procedimentos adotados para evitar o agravamento do quadro clínico do paciente. Foi aplicado um questionário, com oito perguntas abertas e fechadas. Os resultados foram analisados segundo as categorias tempo de formação e especialidade. Foram entrevistados $20,40 \%$ dos profissionais cadastrados no Conselho Regional de Odontologia de Santa Catarina (CROSC). Verificou-se que a maioria dos entrevistados faz a anamnese já no primeiro contato com o paciente e que as doenças crônicas mais frequentes são diabetes e as cardiovasculares. Concluiu-se que os cirurgiões-dentistas entrevistados valorizam a realização da anamnese criteriosa. Palavras-chave eXame ClínICO - DOENÇAS CRÔNICAS ODONTOLOGIA.
Doutora em Medicina Veterinária pela USP - Disciplinas de Patologia Geral e Patologia Aplicada - Curso de

Odontologia daUniversidade do Vale do Itajaí - Unival

Ms. Materiais Dentários - Disciplinas de Metodologia da Pesquisa e Trabalho de Iniciação Científica dos Cursos de Odontologia e Medicina da Universidade do Vale do Itajaí

- UNIVALI

\author{
Cirurgião-Dentista Universidade \\ do Vale do Itajaí - UnivaLI \\ Cirurgiã-Dentista \\ Universidade do Vale do Itajaí - Univali \\ Cirurgiã-Dentista \\ Universidade do Vale do Itajaí - UnIVALI
}

\section{Abstract}

The medical history is taken in the first contact between the dentist and his/her patient. The information obtained must be kept in a clinical record. Due to the increase in the number of patients from different age groups requiring different treatments, clinical records must be standardized. Considering the importance of this step in dental care, this study sought to check if anamnesis is part of the surgeon-dentists' routine, what are the most frequent systemic and emergency diseases and what are the procedures followed to avoid the patients' clinical worsening. A survey with eight open and closed questions was applied to $20.40 \%$ of the dentists registered in the Regional Council of Dentistry of Santa Catarina (CROSC). The results were analyzed according to the categories of training time and expertise. It was found that most respondents took the medical history already in the first contact with the patient and the most common chronic illnesses are diabetes and the cardiovascular diseases. Results showed that the interviewed dentists value the procedure of taking solid medical histories.

KEYWORDS CLINICAL EXAM - CHRONIC DISEASES DENTISTRY. 


\section{INTRODUÇÃO}

Atualmente, os cursos de graduação na área de Odontologia estão enfatizando a aplicação de uma anamnese como parte inicial do exame clínico odontológico e a utilização correta de fichas clínicas, bem como seu adequado arquivamento, porque estes registros constituem um instrumento fundamental na avaliação do padrão de qualidade do atendimento e na defesa nos casos judiciais. A elaboração de fichas clínicas e sua conservação em arquivo próprio são deveres dos profissionais conforme o artigo do Código de Ética Odontológica. ${ }^{1}$

A anamnese é feita no primeiro contato entre o cirurgião-dentista e o paciente antes da realização do exame físico, quando este relata sua história médica e da doença atual com suas próprias palavras, e o profissional deve extrair o máximo de informações relevantes. O profissional deve transferir cuidadosamente essas informações para uma ficha clínica e, se necessário, realizar perguntas adicionais. ${ }^{2}$

O prontuário odontológico é um documento singular para o conhecimento, a qualquer tempo, do diagnóstico e tratamento realizados, assim como do prognóstico e eventuais intercorrências, além de ser o mais perfeito instrumento de defesa. ${ }^{3}$ A cada dia é visível a necessidade de se padronizar as fichas clínicas, uma vez que o número de pacientes necessitando de tratamentos especiais vem crescendo em indivíduos de diferentes faixas etárias. Aumentou o número de pacientes com doenças sistêmicas, sob medicação, idosos com dentes necessitando de uma odontologia adequada à sua situação, além do aumento das técnicas invasivas na odontologia, que implicam, também, um aumento do estresse no paciente e podem agravar uma doença sistêmica preexistente. Os problemas de saúde modificam-se com o decorrer dos anos. O envelhecimento leva a várias alterações fisiológicas em todo o organismo, havendo uma grande prevalência de doenças crônicas nestes indivíduos, que constituem a maior parcela de pessoas que necessitam de atendimento nos serviços de saúde. ${ }^{4}$ Em virtude desta preocupação, propôs-se uma pesquisa com cirurgiõesdentistas da cidade de Itajaí-SC, com o intuito de verificar se a anamnese integra a rotina destes profissionais, quais doenças crônicas sistêmicas e emergenciais são mais encontradas no consultório, e quais os procedimentos adotados para evitar o agravamento do quadro clínico dos pacientes.

\section{MATERIAIS E MÉTODOS}

A população-alvo era composta pelos 245 cirurgiões-dentistas do município de Itajaí cadastrados no Conselho Regional de Odontologia de Santa Catarina. ${ }^{5}$ Desta população, foi constituída uma amostra não probabilística, obtida por conveniência, com um total de 50 sujeitos, ou seja, $20,40 \%$ da população-alvo. Para estes cirurgiões-dentistas, foram explicitados os objetivos da pesquisa e, em caso de recusa, um novo profissional era procurado. $\mathrm{O}$ instrumento de coleta de dados foi um questionário composto por oito questões, entre abertas e fechadas, aplicado diretamente por dois pesquisadores (Anexo 1). A partir das respostas foram estruturadas as categorias de análise. O trabalho foi aprovado pela Comissão de Ética em Pesquisa da Univali, com parecer número 534/2005.

A análise foi realizada mediante tabulação dos dados e cálculos de estatística descritiva (distribuição de frequência relativa e absoluta), levando-se em conta o tempo de formado e a especialidade. Para o tempo de formado, foram constituídas três classes, a saber: formados até cinco anos, formados entre cinco anos e um mês até quinze anos, e formados há mais de quinze anos. Para a categoria especialidade, foram consideradas as seguintes classes: clínica geral, ortodontia, prótese, periodontia, endodontia, cirurgia e implantes, dentística restauradora, odontopediatria e outras especialidades.

\section{Resultados}

A caracterização da amostra encontra-se na tabela 1.

Para critério de comparação das respostas foi utilizada a categoria tempo de formação dos profissionais. Quando estes foram questionados sobre se possuíam fichas com identificação de seus pacientes obtivemos um total de $100 \%$ de respostas afirmativas em todos os grupos.

Da mesma forma, quando questionados sobre a realização da anamnese na primeira consulta, apenas no grupo com tempo de formação de 5 a 15 anos houve uma resposta negativa. Nos demais grupos todas as respostas foram afirmativas. 


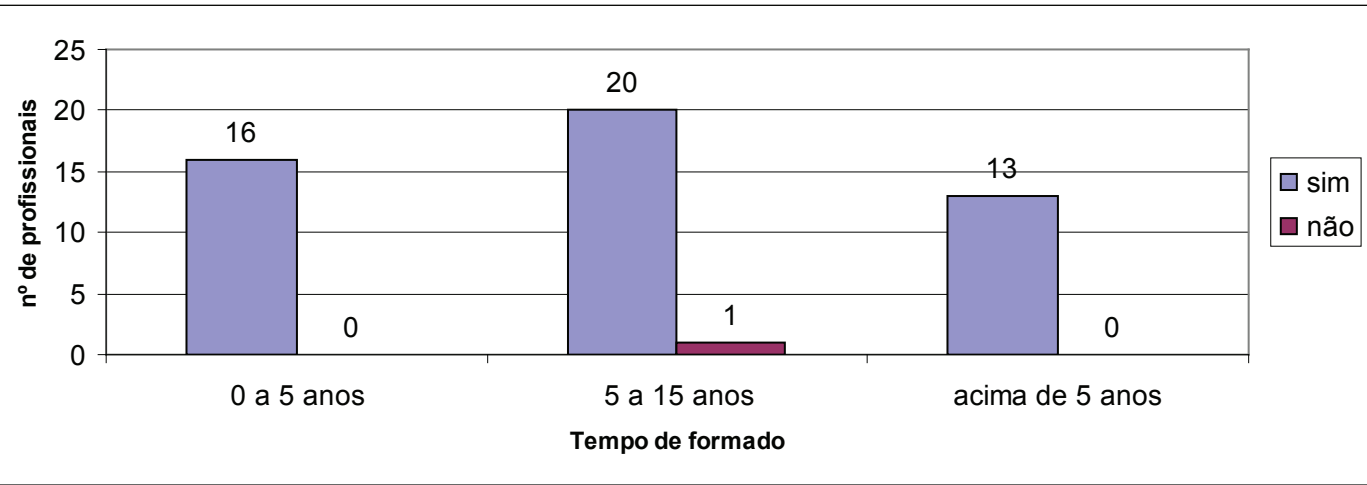

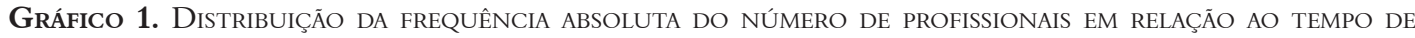
FORMADO SEGUNDO A REALIZAÇÃO DE ANAMNESE NA PRIMEIRA CONSULTA ODONTOLÓGICA.

Quando questionados sobre os procedimentos adotados na rotina do tratamento odontológico, a anamnese criteriosa do paciente foi a atitude mais tomada, seguida pela profilaxia antibiótica.

Foram citadas inúmeras doenças sistêmicas crônicas apresentadas pelos pacientes dos profissionais entrevistados. Nossos resultados mostraram que as doenças crônicas sistêmicas mais encontradas nos pacientes dos profissionais entrevistados são diabetes, cardiopatias e hipertensão.

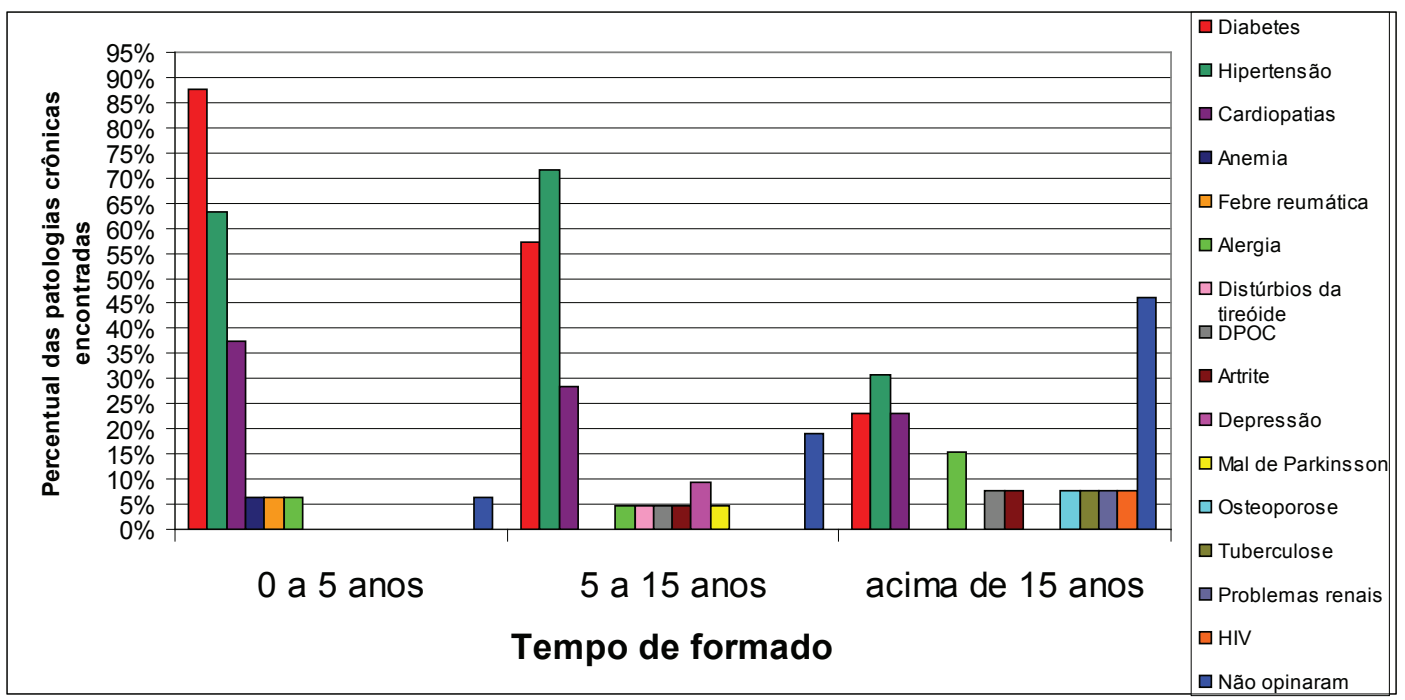

Gráfico 2. Distribuição do NúMERO DE PROFISSIONAIS EM RELAÇÃO AO TEMPO DE FORMADO SEGUNDO AS DOENÇAS CRÔNICAS SISTÊMICAS MAIS ENCONTRADAS NOS PACIENTES.

No que se refere às emergências ocorridas nos consultórios odontológicos em virtude de doenças presentes nos pacientes, o número de ocorrências no grupo formado até 5 anos foi de 3 casos; já no grupo de 5 a 15 anos foram de 9 casos relatados, enquanto no grupo com maior tempo de formação foi de 2 casos. 
Percentual das ocorrências

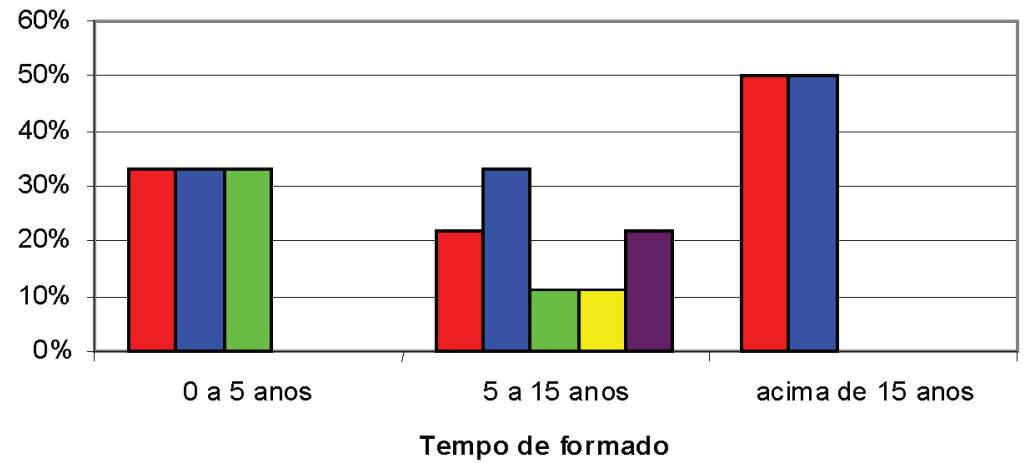

$\square$ Lipotimia
$\square$ Crise hipertensiva
$\square$ Epilesia
$\square$ Dist. Gastrointestinais
$\square$ Alergia

Gráfico 3. Distribuição do percentual de profissionais em relação ao tempo de formado, Segundo as EMERGÊNCIAS JÁ ATENDIDAS EM SEU CONSULTÓRIO ODONTOLÓGICO.

Quando os profissionais foram questionados sobre a possibilidade de o estado de saúde de seus pacientes interferir no plano de tratamento odontológico de rotina, todos os profissionais formados até 5 anos responderam afirmativamente à pergunta, enquanto nos grupos de $5 \mathrm{a} 15$ anos de formados e acima de 15 anos, a resposta foi negativa. Quando perguntados sobre a interferência do estado geral do paciente na execução do plano de tratamento odontológico, os cirurgiões-dentistas responderam, em sua maioria, que o estado de saúde de seus pacientes define o plano de tratamento; a segunda resposta mais apresentada foi que os problemas sistêmicos podem gerar alterações na cavidade bucal.

Em relação ao questionamento sobre a possibilidade de o tratamento odontológico agravar uma condição patológica preexistente no paciente, obtivemos apenas uma resposta negativa, no grupo com menos tempo de formação, e duas respostas negativas nos grupos com maior tempo de formação ( 5 a 15 anos e acima de 15 anos). Houve resposta idêntica quando questionados sobre quais os procedimentos adotados para evitar o agravamento da doença.

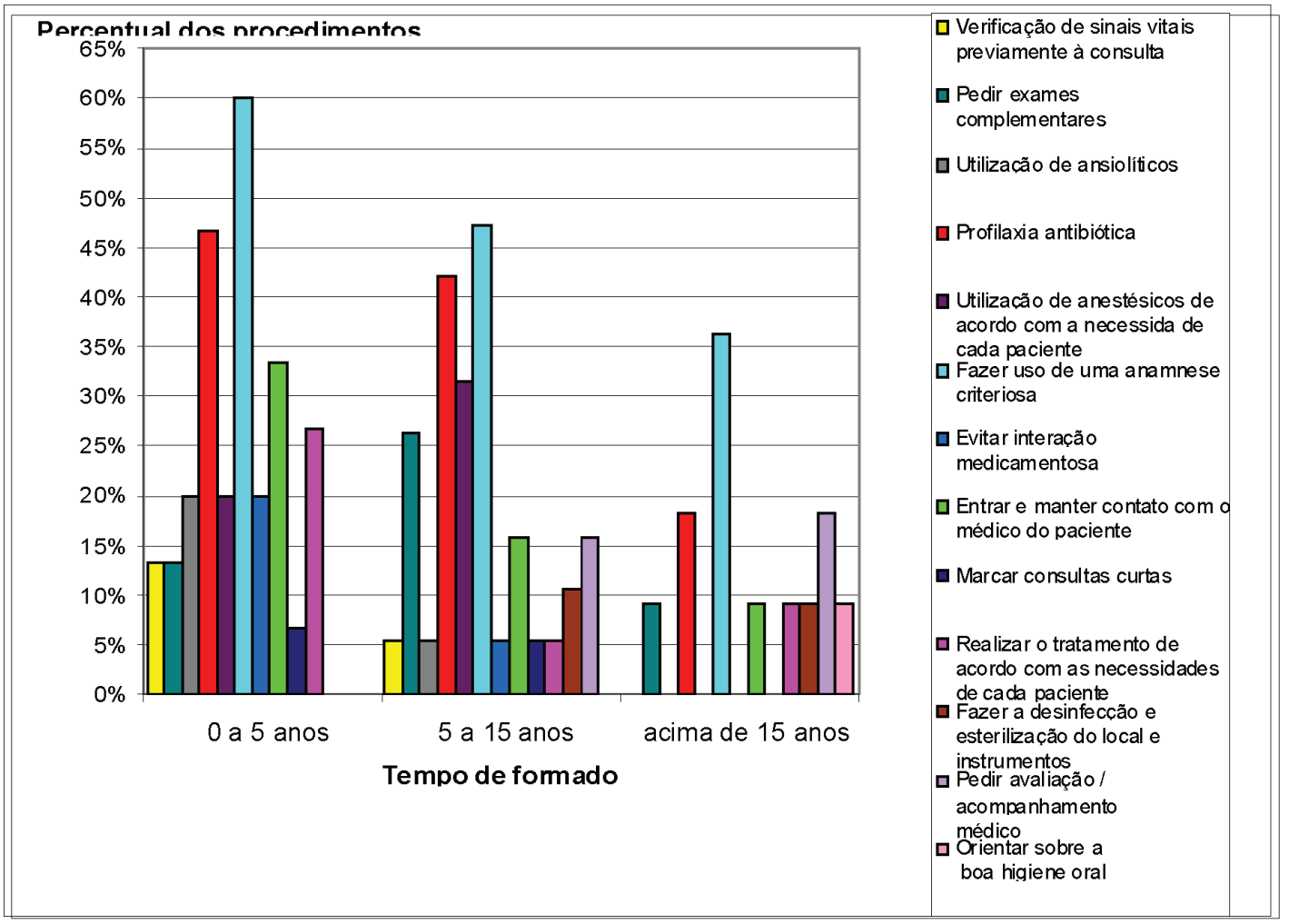

Gráfico 4. Distribuição do PERCENTUAL de PROFisSionais EM RELAÇÃo AO TEMPO DE FORMAdO, SEGUNDO OS PROCEDIMENTOS ADOTADOS PELO CIRURGIÃO-DENTISTA PARA EVITAR O AGRAVAMENTO DO QUADRO CLÍNICO DO PACIENTE. 
O número de pacientes acima de 60 anos atendidos nos consultórios odontológicos ainda é baixo.

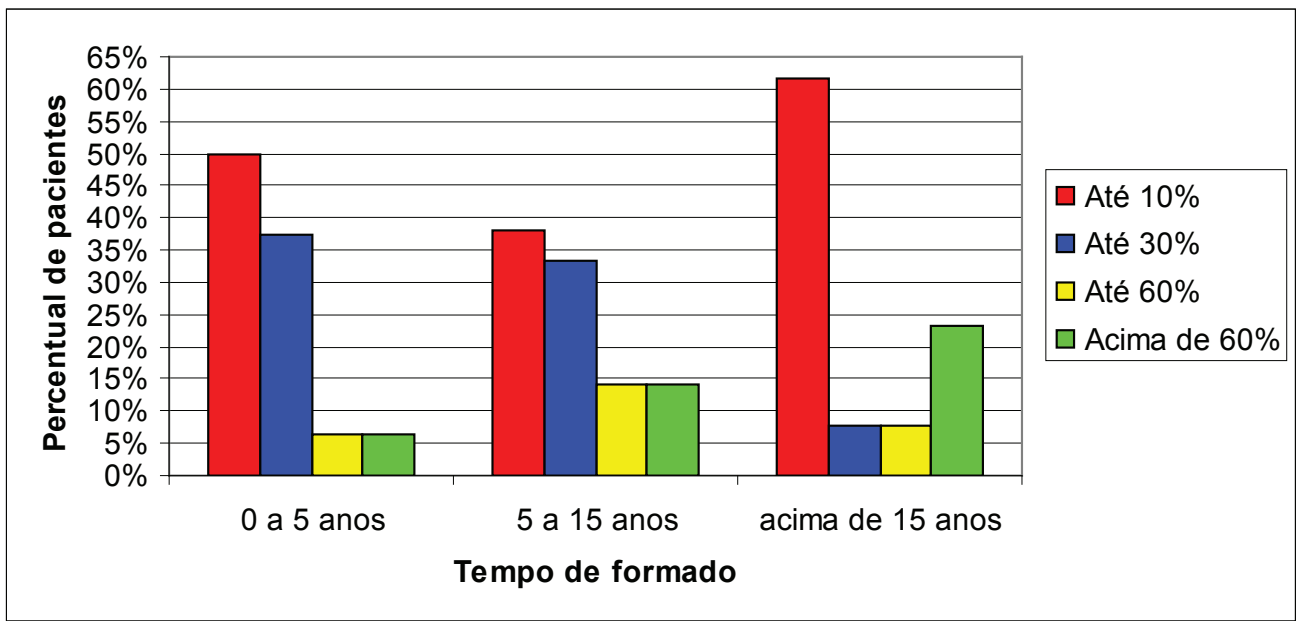

Gráfico 5. Distribuição do PERCENTUAL de PACIENTES ACIMA DE 60 ANOS SEGUNDO O TEMPO DE FORMADO.

\section{DISCUSSÃO}

No estudo de Loeppky e Sigal, ${ }^{6}$ do total dos cirurgiões-dentistas abordados, $52 \%$ não responderam ao questionário, mostrando, assim, que nossa população foi mais receptiva ao nosso questionário do que a do estudo em questão. A orientação sobre as doenças que ocorrem nos órgãos e sistemas do corpo humano deve ser passada ao cirurgião-dentista durante seu período de graduação; se isso não ocorrer, ele pode se sentir inseguro ao responder sobre o estado de saúde geral de seu paciente. Aproximadamente um terço dos entrevistados não respondeu ao questionário, podendo indicar desconhecimento ou descaso por parte deles ou, ainda, medo de se comprometer com sua participação.

Neves $^{7}$ afirmou que alguns profissionais se preocupam apenas em resolver o problema da queixa principal do paciente, descuidando da anamnese por acharem que é muito tempo perdido, e que esse tempo poderia ser utilizado em algo que resultasse em lucro em dinheiro. A elaboração de fichas clínicas e sua conservação em arquivo próprio são deveres dos profissionais, segundo artigo do Código de Ética Odontológica. ${ }^{1}$ O prontuário deve conter as respostas obtidas pelo cirurgião-dentista durante a entrevista, além do planejamento e de todos os procedimentos já realizados no paciente. Todos os nossos entrevistados afirmaram possuir prontuários de seus pacientes.

Em uma pesquisa realizada em Campina
Grande - PB, identificou-se que 100\% dos profissionais elaboravam e guardavam fichas clínicas de seus pacientes, embora apenas $44 \%$ soubessem elaborar corretamente a documentação. Esta pesquisa mostrou também que apenas $38,9 \%$ dos profissionais conheciam as finalidades da documentação, e que as finalidades clínicas e administrativas se sobressaíram sobre a finalidade legal. ${ }^{3}$ Quando corretamente preenchida e armazenada, a documentação odontológica é uma fonte de informações para os pacientes e serve de prova para os profissionais em questões jurídicas.

As doenças que mais levam ao óbito no mundo inteiro são as cardiovasculares. ${ }^{8}$, 9 Também no consultório dos cirurgiõesdentistas entrevistados estas são as doenças crônicas mais frequentes nos pacientes.

A abordagem da saúde geral do paciente, na prática odontológica, vem sendo enfatizada desde a década de 1990. Em 1994, Douglas ${ }^{10}$ já relatava que, a partir do século XXI, os dentistas viriam a se tornar mais próximos dos médicos e das ciências biológicas, em virtude dos avanços nas técnicas diagnósticas de biologia molecular na prevenção, diagnóstico e terapêutica das doenças.

Um estudo realizado com cirurgiõesdentistas canadenses mostrou que mais de $80 \%$ dos cirurgiões-dentistas entrevistados atendem pacientes portadores de alguma doença sistêmica. As mais frequentes foram arritmias, diabetes mellitus, cardiopatias e hipertensão arterial, além de deficiências mentais, como 
síndrome de Down e paralisia cerebral. ${ }^{6}$ Nossos resultados demonstraram que as emergências mais frequentes são relacionadas ao diabetes e aos distúrbios cardiovasculares. Tal fato reforça a ideia de que o uso de anamnese aumenta a confiança do paciente no cirurgião-dentista, diminuindo sua ansiedade e contribuindo para o decréscimo de casos de emergência no consultório odontológico. Assim, pacientes portadores de diabetes e hipertensão arterial devem estar sempre compensados durante todo o tratamento odontológico, evitando emergências. ${ }^{9}$

Além disso, para o cirurgião-dentista evitar situações de emergência no consultório, ele deve conhecer o paciente de forma geral, o que se torna um desafio para estes profissionais já que a grande maioria dos pacientes não se conhece. ${ }^{11} \mathrm{~A}$ avaliação prévia ao tratamento ajuda o profissional no controle de emergências no consultório odontológico. Por exemplo, um diabético pode necessitar de modificações na dieta, na medicação e na duração da consulta. ${ }^{12}$

Muitas vezes são encontradas lesões na cavidade bucal que são manifestações de doenças degenerativas, infecciosas, metabólicas, endócrinas e psíquicas. ${ }^{13,14}$

O cirurgião-dentista deve ter maior responsabilidade durante o tratamento de um paciente com problemas médicos. E, para isso, um bom profissional deve estar capacitado a efetuar uma boa anamnese, incluindo a história médica. ${ }^{15}$

Em algumas condições médicas é aconselhável a realização de profilaxia antibiótica, como no caso da doença nas válvulas cardíacas, endocardites prévias, cirurgias pulmonares com shunts e cardiomiopatias hipertróficas. ${ }^{3}$ Atualmente os cirurgiões-dentistas estão tratando maior número de pacientes idosos portadores de doenças sistêmicas crônicas que tomam um ou mais medicamentos que podem afetar a saúde bucal. A maioria desses idosos ainda preserva a dentição, com necessidades crescentes de trabalhos periodontais e restauradores. ${ }^{4,10}$ Há um número crescente de implantes em pacientes dessa faixa etária.

Nos últimos anos, o número de atendimentos odontológicos a pacientes com idade superior a 60 anos tem aumentado. Com o aumento da longevidade da população, as doenças sistêmicas e crônicas e o uso de múltiplos medicamentos devem aumentar. Estes dados indicam a necessidade de $\mathrm{o}$ cirurgião-dentista estar mais preparado para o atendimento desta população, bem como estar atento a um detalhado procedimento de exame clínico. $^{16}$

O cirurgião-dentista deve ter maior responsabilidade durante a realização do tratamento de um paciente com problemas sistêmicos. Para isso, o bom profissional deve ser capaz de realizar uma boa investigação do paciente, incluindo a anamnese e o exame físico. O profissional deve também conhecer as implicações das doenças sistêmicas nos tratamentos dentários, ter sabedoria para prevenir complicações médicas e ser capaz de estabelecer vínculos entre estado geral e doenças orais. ${ }^{15}$

\section{Conclusão}

Os cirurgiões-dentistas entrevistados mostraram que têm conhecimento e controle dos pacientes por possuírem um prontuário clínico e realizarem a anamnese.

As doenças crônicas sistêmicas mais encontradas pelos profissionais entrevistados foram: hipertensão, diabetes e cardiopatias.

Os tipos de emergências mais encontrados estão relacionados com alterações cardiovasculares.

Dentre os procedimentos adotados para evitar o agravamento do quadro clínico dos pacientes, o mais citado entre os profissionais foi o uso de anamnese criteriosa.

\section{REFERÊNCIAS BibLIOGRÁFICAS}

1. Carvalho GP. Prontuário odontológico incompleto ou ausente: o que pode acontecer? Saúde. 2002; 16 (01): 55-9.

2. Beltrán R. Diagnóstico: base da prática odontológica. [periódico on-line] 2005 Acesso em: 2005 Oct 1. Disponível em: URL: <http://www.jornaldosite.com.br/ arquivo/anteriores/beltran/beltran27p. htm >.

3. Maciel ML, Xavier YM, Leite PH, Alves PM. A documentação odontológica e a sua importância nas relações de consumo: um estudo em Campina Grande - PB. Pesq Bras Odontopediatr Clin Integr. 2003; 3(2): 53-8.

4. Silva AL, Saintrain MVL. Interferência do perfil epidemiológico do idoso na atenção odontológica. Rev. Bras. Epidemiol. 2006; 9: 242-250.

5. CROSC. Profissionais por município. 2007. Acesso em: 2007 Mar 22. Disponível 
em: URL: <http://www.crosc.org.br>.

6. Loeppky WP, Sigal MJ. Patients with special health care needs in general and pediatric dental practices in Ontario. J Can Dent Assoc. 2006/2007; 72(10): 915a-915f.

7. Neves MIR. Importância da anamnese para o diagnóstico, prognóstico e tratamento das enfermidades da cavidade bucal. Medcenter [periódico on-line]. 2001 Out. [Acesso em: 2005 Set 6]. Disponível em: <http://www. odontologia.com.br/artigos.asp?id $=46>$.

8. OMS. Relatório conjunto dos especialistas da OMS/FAO em dieta, nutrição e prevenção de doenças crônicas. 2005. [Acesso em: 2007 Mar 30]. Disponível em: $<$ http://www.paho.org/Portuguese/HCP/ HCV/rimsa13-20-p.pdf>.

9. Manetta EC, Brunetti RF, Montenegro, FLB. Odontogeriatria: uma nova perspectiva de trabalho. J Bras Odontol Clín. 1998; 2(10): 85-7. [cited 2007 Mar 24]. Available from URL: <http://www.odontologia.com. br/artigos.asp? $\mathrm{id}=481 \&$ idesp $=19 \&$ ler $=\mathrm{s}>$.

10. Douglas CW. Is dental practice science based? J Am Coll Dent 1994; 61 (2).13-6.

11. Haidámus I. Emergências no consultório odontológico. [Periódico on-line][cited 2007 Mar 22]. Disponível em: < http://www. jornaldosite.com.br/arquivo/anteriores/ artivan1.htm>.

12. Sonis ST, Fazio RC, Fang L. Princípios e prática de medicina oral. Rio de Janeiro: Guanabara Koogan; 1985. p. 497.

13. Araújo CI de. Atenção integral em saúde ao idoso: uma realidade brasileira? MedCenter. 2007 Mar. [cited 2007 Mar 24]. Available from URL: <http:// www.odontologia.com.br/artigos. asp?id $=$ 704\&idesp $=19 \&$ ler $=\mathrm{s}>$.

14. Arita A, Arita O, Keppe NR. Importância da prevenção na odontologia para a saúde do corpo. Doctor Life. Available from URL: $<$ http://www.doctorlife.com.br/artigo. asp?IdArtigo $=3>$. Acesso em: 2005 Sep 5.

15. Abraham-Inpijn L. Anestesia local em pacientes apresentando patologias médicas, utilidade da anamnese na prevenção de complicações médicas no consultório odontológico. Rev Belge Med Dent. 2000;55 (1):72-79.

16. Kreuger MRO, Pedrini RD, França F, Diegoli $\mathrm{N}$. Índice de salivação correlacionado a idade e a presença de patologias sistêmicas em idosos frequentadores do centro de convivência do idoso no município de Itajaí (SC). Rev Odontol Unesp. 2009; 38:53-8.

Recebimento: 6/7/2010 Aprovação: 26/4/2011

\section{Anexos}

Anexo 1: Modelo do questionário aplicado aos cirurgiões-dentistas.

Dados de identificação:

Tempo de profissão:

Especialidade:

Dados complementares:

1. Possui ficha com dados de identificação do paciente? ( ) sim ( ) não

2. É realizada anamnese do paciente na primeira consulta? ( ) sim ( ) não

3. Quais as patologias crônicas sistêmicas mais frequentes entre seus pacientes?

4. Já atendeu alguma situação de emergência em seu consultório odontológico devido a alguma outra patologia presente em seu paciente?

( ) $\operatorname{sim}($ ) não

Qual (is)?

5. Acha que o estado de saúde de seu paciente pode interferir no plano odontológico de rotina? ( ) $\operatorname{sim}($ ) não Por quê?

6. Acha que o tratamento odontológico pode agravar uma condição patológica sistêmica preexistente em seu paciente? ( ) sim ( ) não Quais procedimentos são adotados para evitar o agravamento da doença?

7. Qual a frequência de pacientes acima de 60 anos atendidos em seu consultório por ano? 
8. Considera que aumentou o número de pacientes de terceira idade nos últimos anos em seu consultório? ( ) sim ( ) não

LISTA DE TABELAS

Tabela 1. Profissionais entrevistados Divididos por Categorias

\begin{tabular}{l|l|c|c}
\hline & CATEGORIAS & N $^{\circ}$ DE CDs & $\%$ \\
\hline \multicolumn{1}{l|}{ Tempo de formação } & & 16 & 32 \\
\hline & a a 5 anos 15 anos & 21 & 42 \\
\hline & acima de 15 anos & 13 & 26 \\
\hline Local de formação & & 58 \\
\hline & Univali & 29 & 42 \\
\hline & Outros & 21 & \\
\hline Sexo & & & 58 \\
\hline & Masculino & 29 & 42 \\
\hline & Feminino & 21 & 44 \\
\hline Especialidades & Clínica geral & & 18 \\
\hline & Ortodontia & 22 & 10 \\
\hline & Prótese dentária & 9 & 8 \\
\hline & Cirurgia/implante & 5 & 6 \\
\hline & Endodontia & 4 & 4 \\
\hline & Periodontia & 3 & 4 \\
\hline & Dentística restauradora & 2 & 4 \\
\hline & Odontopediatria & 2 & 2 \\
\hline & Pacientes especiais & 2 & \\
\hline
\end{tabular}

